\title{
Inkjet Printing of 2D Layered Materials
}

\author{
Jiantong $\mathrm{Li},{ }^{*[a]}$ Max C. Lemme, ${ }^{[a, b]}$ and Mikael Östling ${ }^{[a]}$
}

Inkjet printing of $2 \mathrm{D}$ layered materials, such as graphene and $\mathrm{MoS}_{2}$ has attracted great interests for emerging electronics. However, incompatible rheology, low concentration, severe aggregation and toxicity of solvents constitute critical challenges which hamper the manufacturing efficiency and product quality. Here, we introduce a simple and general technology concept (distillation-assisted solvent exchange) to efficiently overcome these challenges. By implementing the concept, we have demonstrated excellent jetting performance, ideal printing patterns and a variety of promising applications for inkjet printing of $2 D$ layered materials.

\section{Introduction}

In everyday life, inkjet printing is a well-known technique which produces texts and graphics by ejecting ink droplets through nozzles onto paper, plastic, or other substrates. Inkjet printers are among the most common facilities used in office and household. ${ }^{[1]}$ Recently, the applications of inkjet printing have been extended to manufacturing intelligent components and smart systems for organic and printed electronics, such as organic light-emitting diodes, displays, thin film transistors, memories, batteries and micro-electromechanical systems. ${ }^{[2-4]}$ Inkjet printing possesses several attractive features, including direct (mask-free) patterning, purely additive operation, compatibility with versatile materials and flexible substrates, low waste of materials, cost efficiency, and scalability to large area manufacturing. ${ }^{[5]}$ In addition, highresolution inkjet printing techniques have been developed with critical dimensions as small as $1 \mu \mathrm{m} .{ }^{[5,6]}$ All of them make inkjet printing very promising for emerging electronics.

In new applications, advanced inks based on conducting/ semiconducting polymers ${ }^{[1]}$ and functional nanoparticles ${ }^{[7,8]}$ are often formulated to realize the functionality. Recent research shows great interest in two-dimensional (2D) layered materials, such as graphene, $\mathrm{MoS}_{2}$, and $\mathrm{WS}_{2} \cdot{ }^{[-11]}$ These materials comprise single or few layers of atoms and hence integrate a number of unique properties. For instance, graphene (a layer of carbon atoms) is electrically and thermally conductive, optically transparent, and mechanically flexible. Moreover, the nature of the 2D atomic crystals produces extremely high specific surface area, which is fascinating for energy storage applications. In particular, these materials are compatible with liquid-phase fabrication. A variety of techniques has been developed to prepare liquid dispersions of the $2 \mathrm{D}$ materials ${ }^{[11]}$ as well as their derivatives such as graphene oxide. ${ }^{[12]}$ This merit attracts great interest to combine the unique $2 \mathrm{D}$ materials with the promising inkjet printing technique for novel applications. ${ }^{[9,10]}$
In spite of considerable efforts in inkjet printing of $2 \mathrm{D}$ materials (especially graphene), several challenges slow down progress in the printing performance, efficiency and reliability, including: (1) Most inkjet printers favor a suitably low ink viscosity around $10 \mathrm{cP}$. However, excellent solvents for dispersing 2D materials, such as dimethylformamide (DMF) and $\mathrm{N}$ methylpyrrolidone (NMP), ${ }^{[11]}$ have too low viscosity $(<2 \mathrm{cP})$. Such incompatible rheology may severely degrade the jetting performance. (2) Usually the concentration of as-prepared 2D material dispersion is very low $(<0.1 \mathrm{mg} / \mathrm{mL})$. This is not efficient for inkjet printing since tens of printing passes are often needed to obtain functional films. (3) The 2D materials easily aggregate in the dispersions. This results in big particles which may block the nozzles and hence influence the jetting reliability. (4) DMF and NMP are toxic, which causes environmental concerns when they are adopted for mass production in industry.

Recently, we developed a simple and general technology ${ }^{[13,14]}$ to formulate advanced inks for 2D materials. It is mainly based on a distillation-assisted solvent exchange technique. ${ }^{[15]}$ This Concept paper elaborates the technology aiming to facilitate its employment and extension in future studies.

[a] Dr. J. Li, Prof. M. C. Lemme, Prof. M. Östling KTH Royal Institute of Technology

School of Information and Communication Technology

Electrum 229, SE-164 40 Kista, Sweden

Fax: (+46) 87904300

E-mail: jiantong@kth.se

[b] Prof. M. C. Lemme

Graphene-based Nanotechnology

University of Siegen

Hölderlinstr. 3, 57076 Siegen, Germany 
Dr. Jiantong Li received his B. Sc. from University of Science and Technology of China in 2002, his M. Sc. from Shanghai Institute of Ceramic, Chinese Academy of Science in 2005, and his Ph.D. from KTH Royal Institute of Technology (Sweden) in 2010. Since then, he has been a senior researcher at KTH. His research interest includes printed electronics, inkjet printing, carbon nanotubes, graphene and its analogues, energy storage, and Monte Carlo simulations for percolation theory.

\section{Prof. Max Christian Lemme received} his Dipl.-Ing. and PhD degrees from RWTH Aachen University, Germany in 1998 and 2004. He is HeisenbergProfessor for Graphene-based Nanotechnology at the University of Siegen, Germany. Until 2008 he was Head of Technology at nanotechnology start-up AMO $\mathrm{GmbH}$ in Germany. From 2008 to 2010 he was a Humboldt Research Fellow at Harvard University and from 2010 -

2012 he was Guest Professor at KTH Royal Institute of Technology. His research interests are in nanoelectronic devices, in particular based on graphene and related $2 D$ materials and their co-integration with silicon technology. Lemme was awarded a young investigator grant (NanoFutur) from the German Ministry for Education and Research for work on graphene in 2006 and an ERC starting grant in 2012.

\section{Prof. Mikael Östling received his PhD} degree from Uppsala University, Sweden in 1983. He holds a position as professor in solid state electronics at KTH Royal Institute of Technology. Between 2004 and 2012 he was Dean of the School of Information and Communication Technology, KTH. His research interests are within integrated devices and circuits. Östling was a senior visiting Fulbright

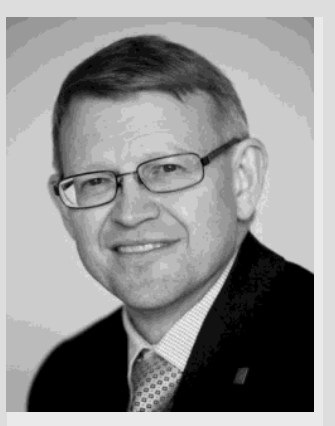
Scholar 1993-94 with the Center for Integrated Systems at Stanford University, and a visiting professor with the University of Florida, Gainesville. In 2005 he co-founded the company TranSiC which was successfully acquired in full by Fairchild Semiconductor. In 2009 he received the first ERC award for advanced investigator grant. He is an editor of the IEEE Electron Device Letters and a Fellow of the IEEE.

\section{Solvent Exchange for Ink Formulation}

The biggest challenge for inkjet printing of $2 \mathrm{D}$ layered materials is that there are no common solvents which excel in exfoliating $2 \mathrm{D}$ materials and simultaneously possess compatible rheology for inkjet printing. To address it, our technology concept is fairly simple: The 2D materials are first exfoliated in a suitable solvent and then the solvent is exchanged with another solvent which is compatible with inkjet printing. With this concept, it is not necessary to seek a common solvent compatible with both exfoliation and inkjet printing. As illustrated in Scheme 1, our technology mainly includes four steps:

(1) Raw 2D materials (in bulk form) are exfoliated into single- or few-layer nanosheets in an appropriate solvent (hereafter referred to as exfoliation solvent) through the well-established liquid-phase exfoliation technique. ${ }^{[11]}$

(2) Compatible polymers are added to prevent the acquired 2D nanosheets from restacking and hence stabilize the dispersion. In general, these polymers should be removed after printing.

(3) Another miscible solvent (hereafter referred to as printable solvent) is added into the dispersion. As compared with the exfoliation solvent, the printable solvent has a higher boiling point, higher viscosity, lower volume and lower toxicity. Then, the exfoliation solvent is distilled off and the $2 \mathrm{D}$ nanosheets transfer into the printable solvent.

(4) The new dispersion is tailored with a third solvent (hereafter referred to as tailoring solvent) to attain compatible rheology for inkjet printing.

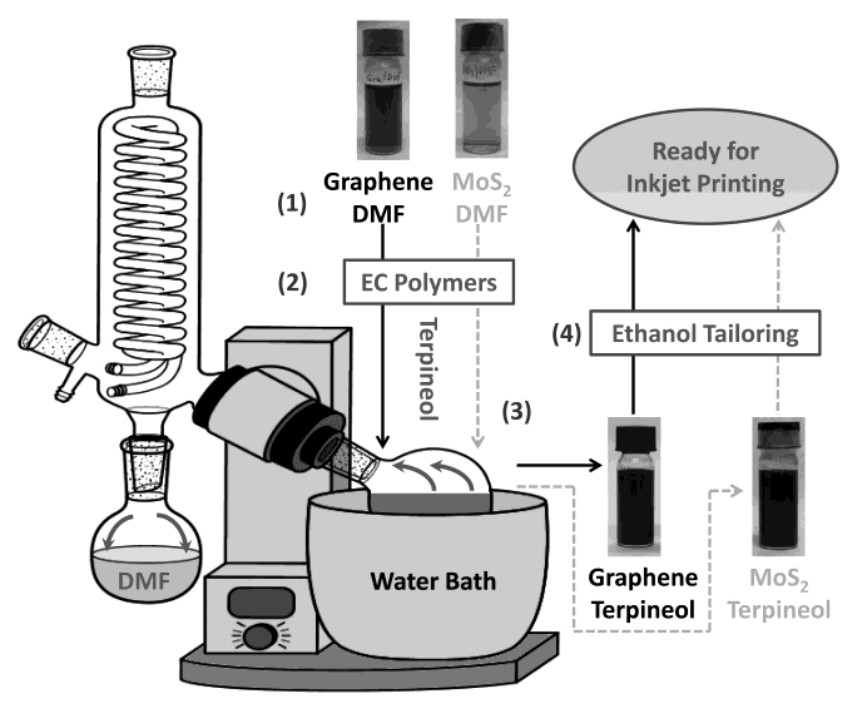

Scheme 1. Schematic illustration of our ink formulation concept for inkjet printing of 2D layered materials, such as graphene and $\mathrm{MoS}_{2}$. The technology includes four steps (see the text) and is mainly based on the distillation-assisted solvent exchange technique (Step 3 ).

We propose the technology described above as a general route to overcome or diminish the challenges in inkjet printing of 2D materials. In terms of different applications, one may implement the concept in different ways. Here we introduce our recent implementation which applies to inkjet printing of graphene ${ }^{[13]}$ and $\mathrm{MoS}_{2} \cdot{ }^{[14]}$ Because of the similarity between graphene and many other $2 \mathrm{D}$ materials, ${ }^{[9-11]}$ we expect that this implementation applies to most 2D layered materials. We also hope that new implementations can be developed in future studies to further extend the technology concept. 


\subsection{Liquid-Phase Exfoliation}

High-quality (nearly defect-free) graphene ${ }^{[16]}$ and other 2D nanosheets ${ }^{[11]}$ have been directly obtained from a simple and general liquid-phase exfoliation technique. Bulk materials (usually powders of the 2D layered materials) are first mixed with an appropriate organic solvent and then exfoliated into 2D nanosheets with the assistance of ultra-sonication. After centrifugation, large particles sediment and the harvested supernatant contains mostly single- or few-layer 2D nanosheets. Systematic studies indicate that the concentration, flake dimension and thickness strongly depend on sonication power, sonication time, and centrifugation rate. ${ }^{[16-19]}$ In general, high sonication power or long sonication time produces high concentration but also introduces more defects. High centrifugation rate gives rise to smaller and thinner nanosheets.

Considering the preference of inkjet printing and the requirement of our technology concept, we choose DMF as the exfoliation solvent. DMF is an excellent and common solvent for exfoliating 2D materials. ${ }^{[11]}$ In particular, as compared with many other exfoliation solvents, such as NMP and dimethyl sulfoxide (DMSO), DMF has a relatively low boiling point $\left(\sim 153^{\circ} \mathrm{C}\right)$, and hence provides more space for the selection of the printable solvent, which must have higher boiling point than the exfoliation solvent. In order to acquire high quality (with fewer defects) of the $2 \mathrm{D}$ materials and make a trade-off between concentration and efficiency, we use low-power bath sonication for a moderate sonication time. Since large flakes may block the nozzles during printing, a high centrifugation rate is used to obtain small flake sizes. In detail, a typical procedure to exfoliate the 2D materials in our implementation is as follows. (1) Powders of raw 2D materials are mixed with DMF at the concentration of $\sim 2 \mathrm{mg} / \mathrm{mL}$. (2) The mixture is bath sonicated for $\sim 20-40 \mathrm{~h}$. During sonication, the bath water is refreshed every few hours to retain nearly constant temperature. (3) The obtained dispersion is centrifuged at $\sim 10,000 \mathrm{rpm}$ for $30 \mathrm{~min}$ to sediment the large particles, and the supernatant is harvested. (4) To confirm the removal of large particles, the supernatant is centrifuged again at $\sim 10,000 \mathrm{rpm}$ for $30 \mathrm{~min}$. The final harvested supernatant is a clear DMF dispersion of the 2D materials, as shown in Scheme 1. Usually the flakes in this dispersion have lateral dimensions much less than $1 \mu \mathrm{m}$ and thicknesses less than $10 \mathrm{~nm}$, but the concentration is rather low. For example, it is $\sim 20 \mu \mathrm{g} / \mathrm{mL}$ for graphene/DMF dispersion, and $\sim 5 \mu \mathrm{g} / \mathrm{mL}$ for $\mathrm{MoS}_{2} / \mathrm{DMF}$ dispersion.

\subsection{Polymer Stabilization}

The obtained low-concentration DMF dispersion of 2D material is fairly stable. However, upon being concentrated, the exfoliated $2 \mathrm{D}$ flakes may restack and the stable period is severely shortened. ${ }^{[15]}$ Therefore, stabilizing polymers (or surfactants) are usually introduced to extend the stable period and improve the printing reliability. Here, we take ethyl cellulose $(E C)^{[20]}$ as the stabilizing polymer. The advantage is threefold. First, EC effectively stabilizes the 2D material inks. We have demonstrated that the EC-stabilized graphene ink is stable for at least several weeks at concentrations of over $1 \mathrm{mg} / \mathrm{mL}$, and the EC-stabilized $\mathrm{MoS}_{2}$ ink $(\sim 0.1 \mathrm{mg} / \mathrm{mL})$ is stable for one week. Second, during printing, EC improves the uniformity of the printed patterns. ${ }^{[13,21]}$ Last, after printing, most EC can be readily removed through a simple annealing, and the burnout temperature is relatively low $\left(\sim 400{ }^{\circ} \mathrm{C}\right.$ in air)..$^{[22]}$ After these steps, the performance of the pristine $2 \mathrm{D}$ materials can be almost fully recovered.
In practice, two kinds of EC are used. They have different viscosity, $4 \mathrm{cP}$ and $22 \mathrm{cP}$ (5\% in 80:20 toluene:ethanol, SigmaAldrich), respectively. The low-viscosity EC is added first into DMF dispersions and sonicated for $1 \mathrm{~h}$ to enhance the interaction with the $2 \mathrm{D}$ materials. Then, the high-viscosity EC is added to further stabilize the dispersion, as well as improve the printing pattern uniformity. ${ }^{[13,21]}$ The amount of EC is calculated so that the concentration of each EC after solvent exchange is around 5 $\mathrm{mg} / \mathrm{mL}$. This EC concentration level greatly improves the ink stability and pattern uniformity, but does not leave evident residue after annealing.

\subsection{Distillation-assisted Solvent Exchange}

The function of the solvent exchange process is twofold. One is to make the $2 \mathrm{D}$ material dispersions printable, and the other is to concentrate the inks. In order to exchange the exfoliation solvent with the printable solvent through distillation, the latter should have a much higher boiling point than the former (preferably the difference is $>25{ }^{\circ} \mathrm{C}$ ). In order to concentrate the inks, the printable solvent should have significantly lower volume than the exfoliation solvent.

In our implementation, we choose terpineol as the printable solvent for several reasons. (1) The boiling point of terpineol is $220^{\circ} \mathrm{C}$, significantly higher than that of DMF $\left(153^{\circ} \mathrm{C}\right)$, a desired merit for the distillation. (2) Terpineol has high viscosity ( $40 \mathrm{cP}$ at $20^{\circ} \mathrm{C}$ ) and is compatible with inkjet printing after being tailored. (3) Low-dimensional materials, such as graphene and carbon nanotubes, are relatively stable in terpineol even at high concentration. ${ }^{[15,23]}$ (4) EC has good solubility in terpineol. Therefore, the combination of terpineol and DMF should be an ideal system for the distillation to formulate 2D material inks.

To concentrate the dispersion, before distillation, the ECstabilized 2D material dispersion is mixed with terpineol at a specific volume ratio (DMF: terpineol) of Rv. Then after DMF is distilled off, the dispersion is concentrated by Rv times. The upper limit of Rv seems to be 60:1 for graphene and 20:1 for $\mathrm{MoS}_{2}$ when the sonication time in the first step is $\sim 40 \mathrm{~h}$. Beyond the limit, the stability of the final inks decreases.

In our experiments, a vacuum distillation is used (Scheme 1). The DMF/terpineol mixture is first put into the left flask of a rotary evaporator and then heated to $80^{\circ} \mathrm{C}$ by water bath. When the pressure is reduced to $\sim 30 \mathrm{mbar}$, DMF begins to evaporate and the DMF steam condenses in the right flask. Once DMF is distilled off, the 2D material is transferred to the terpineol dispersion and hence the solvent exchange is accomplished.

\subsection{Ink Tailoring}

The terpineol dispersions of 2D materials are very viscous (viscosity $>40 \mathrm{cP}$ ) and stable for a long time. Such dispersions may be straightforwardly employed for screen printing ${ }^{[24]}$ but iare incompatible with inkjet printing. Shortly prior to inkjet printing, the viscosity should be tailored with the tailoring solvent to be $\sim 10 \mathrm{cP}$ (at the printing temperature). Obviously, the tailoring solvent should have low viscosity, compatible surface tension, and good dissolving capacity with the printable solvent and the stabilizing polymer.

In our implementation, we use ethanol as the tailoring solvent. Ethanol has a very low viscosity $\left(\sim 1 \mathrm{cP}\right.$ at $\left.20^{\circ} \mathrm{C}\right)$, is miscible with 
terpineol, and dissolves the EC polymer well. It can tailor the rheology of the 2D material inks without significantly diluting them. As for the inkjet printer (Dimatix Material Printer, DMP-2800) in our work, the optimal volume ratio for the tailoring is terpineol : ethanol $=3: 1$. After a rough sonication $(\sim 1 \mathrm{~h})$ of the terpineol/ ethanol mixture, stable and high-concentration inks are obtained.

Finally, for the ink composition, we prefer the combination of two solvents (i.e., terpineol and ethanol) to one single solvent. On one hand, just as it is challenging to find a common candidate for both exfoliation solvent and printable solvent, one can hardly impose all the functionalities of the printable and tailoring solvents into one common solvent. On the other hand, during printing, the two-solvent systems may improve the drop wettability and pattern uniformity.

\subsection{Technology Superiority}

It is worth noticing that the above technology is not an exclusive method to implement the concept of solvent exchange. Some other solvent exchange techniques have also been demonstrated for the preparation of high-concentration graphene dispersions, such as filtering ${ }^{[25]}$ or precipitating ${ }^{[21]}$ graphene from the first solvent and redispersing it into the second solvent, and iteratively extracting graphene from the first solvent to the second one by virtue of its dispersibility difference in the two solvents. ${ }^{[20]}$

As for inkjet printing, however, our distillation-assisted solvent exchange technique has several advantages. (1) The technique is very simple since the solvent exchange is accomplished by only one distillation process. (2) The solvent exchange process takes place completely in liquid phase, preventing the restacking of 2D material flakes to the largest extent. (3) Almost all 2D material flakes are transferred into the printable solvents, greatly reducing material waste. (4) Although many applications prefer low-boiling-point solvents, inkjet printing usually favors highboiling-point inks since low-boiling-point inks are volatile and hence easily block the nozzles. Therefore, the distillation-assisted solvent exchange technique ideally suits inkjet printing because of their common preference of high boiling point for the printable solvent.

\section{Results and Discussion}

With proper parameter settings (driving waveform, cartridge temperature, firing voltage and frequency, etc.) in the inkjet printer (DMP-2800), we have demonstrated that our 2D material inks provide excellent jetting performance and produce conformal, uniform and high-resolution patterns. More importantly, they allow simple and efficient fabrication of a variety of electronic devices with good performance. Typically, several printing passes followed by a simple annealing accomplishes the process.

\subsection{Jetting Performance}

Thanks to the compatible rheology and suitable flake size, both graphene and $\mathrm{MoS}_{2}$ ink droplets are well directed and constantly jetted out of all nozzles at an even velocity, as shown in Figure 1. Such reliable jetting performance facilitates parallel printing with multiple nozzles for efficient large-scale production.
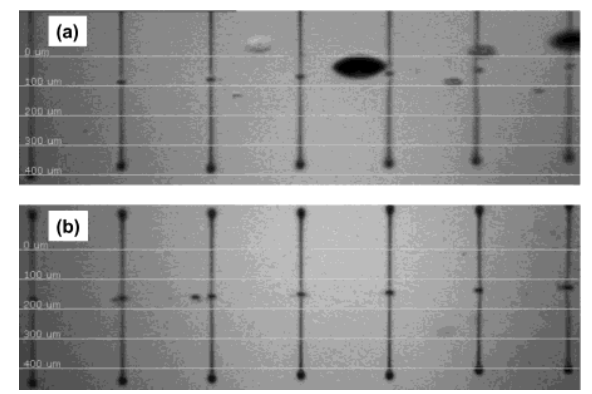

Figure 1. Stroboscopic images of jetted droplets showing the jetting performance of (a) graphene and (b) MoS2 inks. The images were captured using the Drop Watcher system of the DMP-2800 printer in the frozen mode with a strobe delay of $30 \mu \mathrm{s}$. Adapted with permission from Ref. [13] for (a) and Ref. [14] for (b).

\subsection{Printed Patterns}

The excellent jetting performance produces high-quality printing patterns. Figure 2 indicates the printed droplet matrix, lines and films for graphene and $\mathrm{MoS}_{2}$. The as-printed patterns are uniform and conform well to the designs. Without any intentional surface engineering, the resolution (droplet diameter or line width) is 50$80 \mu \mathrm{m}$. In particular, the printed lines are smooth, even, narrow and straight. These are important and indispensable features for future applications in printed electronics. ${ }^{[26]}$

(a)

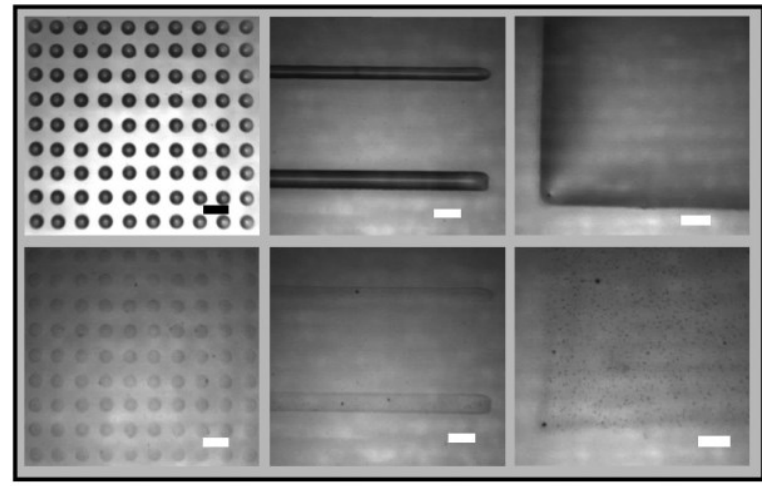

(b)

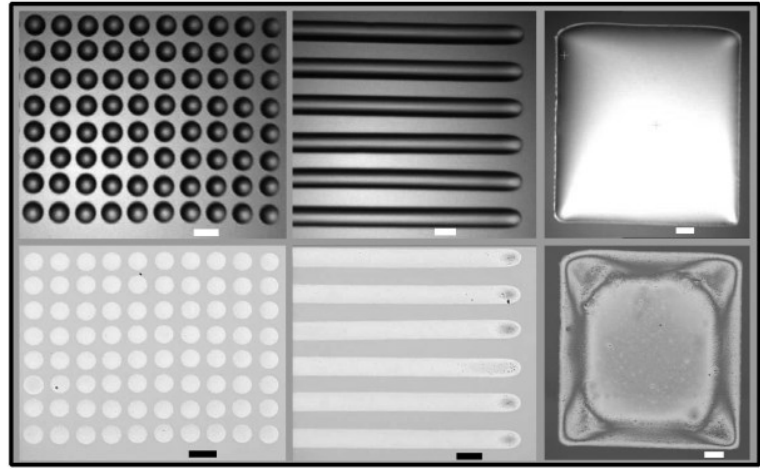

Figure 2. As-printed (upper) and dried (lower) patterns for inkjet printing of (a) graphene and (b) $\mathrm{MoS}_{2}$. All the scale bars are $100 \mu \mathrm{m}$. Adapted with permission from Ref. [13] for (a) and Ref. [14] for (b).

After ink drying, the patterns retain excellent uniformity. Especially for the graphene patterns, the atomic force microscopy (AFM) analyses of the printed droplet and line (Figure 3) quantitatively confirm their uniformity and suggest that they are free from the well-known and usually undesired coffee-ring 
effects. ${ }^{[27]}$ When a spilled drop of coffee (or in general a liquid containing certain solutes) dries on a solid surface, a ring-like deposit along the boundary, rather than a uniform film, is often left. This phenomenon occurs during drying of most liquid solutions/ dispersions. The mechanism is generally ascribed to the pinning of the contact lines (edges) of the drying drops. Since liquid evaporates faster in the edge than in the center, the contact line pinning forces the liquid to flow from the center to replenish the edge. The outward flow carries solutes to the edge and makes it significantly thicker than the center. This causes the coffee-ring effects. For our graphene inks, however, we observe the depinning of contact lines during drying. ${ }^{[13]}$ The reason may be as following. Initially, the ink (terpineol/ethanol mixture) has good wetting on the substrates (Figure 4a). Since ethanol has a very low boiling point $\left(78^{\circ} \mathrm{C}\right)$, it evaporates much faster than terpineol, especially at the contact lines. When ethanol dries, some graphene/EC composite deposits, and the remaining terpineol has poor wetting on this composite surface (Figure 4b). As a result, the poor wetting depins the contact lines and suppresses the coffee-ring effects. However, in contrast to the coffee-ring effects, the de-wetting of terpineol may also cause non-uniformity for the patterns (Figure 4b). Fortunately, this issue can be addressed by increasing the substrate temperature to alleviate the dewetting and expedite the evaporation of terpineol to quickly reach uniform patterns (Figure 4c).
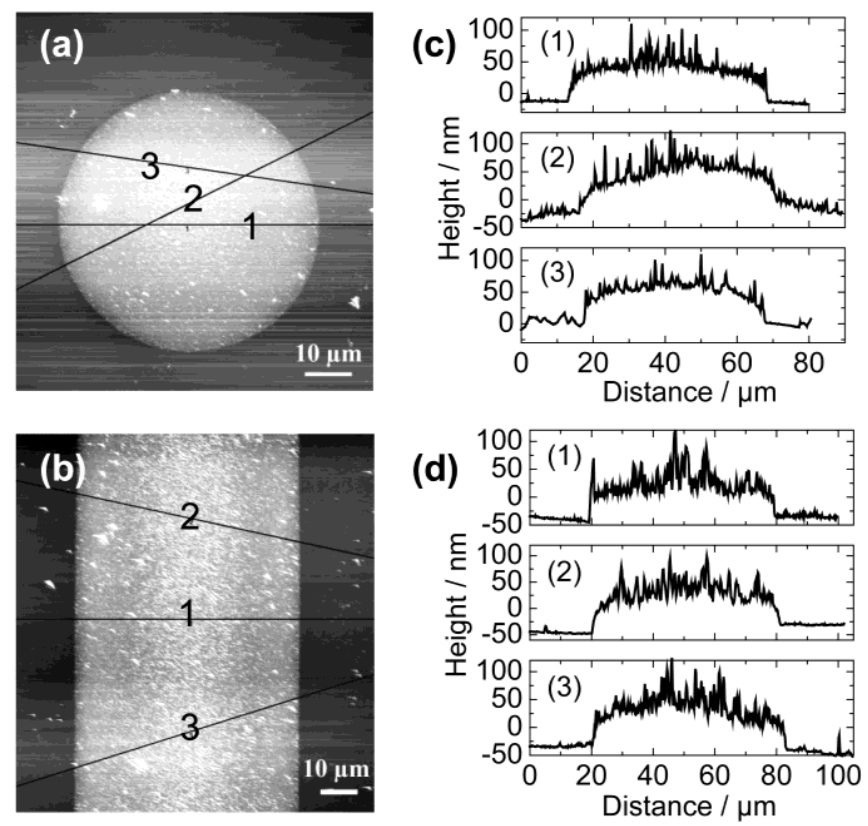

Figure 3. AFM images of a dried droplet (a) and line (b) for the printed graphene patterns in Figure 2a. (c) and (d) are cross-sectional profiles along the three directions in (a) and (b), respectively. The patterns are globally uniform (free from the coffee-ring effects). Adapted with permission from Ref. [13].

For dried $\mathrm{MoS}_{2}$ patterns (Figure 2b), however, the coffee-ring effects are still visible. It might be that the $\mathrm{MoS}_{2} / \mathrm{EC}$ composite has different surface properties than the graphene/EC composite, so that the dewetting of terpineol is not strong enough to depin the contact lines. Consequently, in spite of the great similarity between graphene and $\mathrm{MoS}_{2}$ in liquid-phase processing, we observe their fine distinction in inkjet printing. (a)
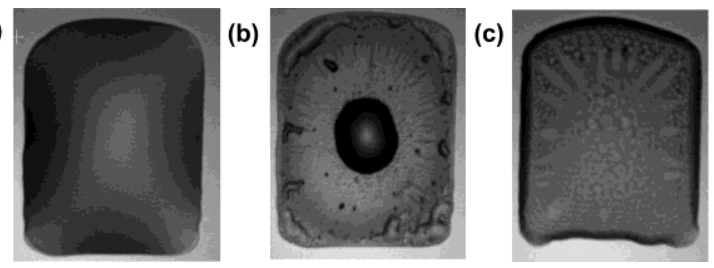

Figure 4. Evaporation processes of inkjet printed graphene films on silicon. (a) Optical micrograph of an as-printed liquid film. (b) The dried pattern with the substrate at room temperature $\left(\sim 25^{\circ} \mathrm{C}\right)$. The dark region in the center consists of the dewetted terpineol. (c) The dried pattern with the substrate at $60{ }^{\circ} \mathrm{C}$ There is no dewetting and the pattern is fairly uniform. Adapted with permission from Ref. [13].

The suppression of coffee-ring effects is of great interest. ${ }^{[28]}$ It should be a new, effective and general strategy to suppress the coffee-ring effects on various substrates through the wettingdewetting transfer in the mixed inks of high- and low-boiling-point solvents and suitable polymer composite, although systematic studies are still needed to further clarify the mechanism. Note the depinning of contact lines is also observed on certain substrates in other studies. ${ }^{[29,30]}$ Besides, in terms of different application requirements, other conventional and novel strategies could also be integrated into our printing technology of 2D materials, such as modifying the particle shape to prevent the outward capillary flow, ${ }^{[31]}$ and mixing different solvents to induce the inward Marangoni flow. ${ }^{[32]}$

\subsection{Device Performance}

Not only should a good inkjet printing technology produce excellent jetting performance and patterns, but it should also retain the performance and functionality of the printed materials. We have used our inkjet printing technology for $2 \mathrm{D}$ materials to print several electronic devices with good performance, such as transparent conductors, supercapacitors and photodetectors.
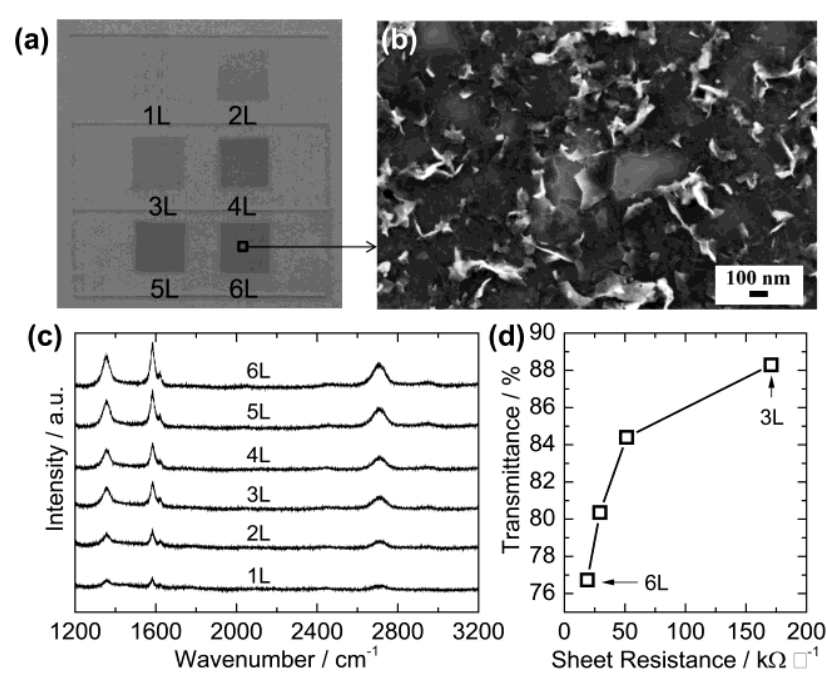

Figure 5. Inkjet printed graphene transparent conductors. (a) Printed graphene square films $(1.5 \mathrm{~cm} \times 1.5 \mathrm{~cm})$ with various printing layers (from 1 to 6 layers) on glass slides. (b) An SEM image of the 6-layer (6L) graphene film. (c) Raman spectra of the printed graphene films. (d) Transmittance (at the wavelength of $550 \mathrm{~nm}$ ) versus sheet resistance for some printed graphene films (3-6 printing layers). Reprinted with permission from Ref. [13] 
Graphene-based transparent conductors. Figure 5 shows printed graphene transparent conductors. The devices are fabricated through printing the graphene inks on glass slides (Figure 6a) followed by a baking process on a hot plate at $400{ }^{\circ} \mathrm{C}$ in air for $1 \mathrm{~h}$. A scanning electron microscopy (SEM) image (Figure 6b) and Raman spectra (Figure 6c) confirm the presence of few-layer graphene flakes without extensive contamination from the EC residue. The transmittance and sheet resistance depend on the number of printing layers (passes). For the device with five printing passes, the transmittance (at a wave length of $\lambda$ $=550 \mathrm{~nm}$ ) is $\sim 80 \%$, and the sheet resistance reaches $30 \mathrm{k} \Omega / \square$. The resistance is about 2-3 orders of magnitude lower than that of other printed graphene devices in the literature. It is also comparable to that of vacuum-filtrated polymer-free graphene films ${ }^{[15]}$ implying that the printing process does not cause severe performance degradation.

Graphene-based supercapacitors. Figure 6 shows inkjet printed micro-supercapacitors in planar (interdigitated) structure on Kapton. The current collectors are printed with commercial silver nanoparticle inks (Figure 6a), while the electrodes are printed using our graphene inks with 8 (Figure 6b) or 4 (Figure $6 \mathrm{c})$ printing layers. From the cyclic voltammetry (CV) curves (Figure 6d-f), the areal-specific capacitances are extracted. With only 8 printing layers of graphene, the device (Figure 6b) possesses a capacitance of $\sim 0.6 \mathrm{mF} / \mathrm{cm}^{2}$ at the scan rate of 100 $\mathrm{mV} / \mathrm{s}$. This value is comparable to that of similar microsupercapacitors made from the laser scribing technique. ${ }^{[33]}$ In particular, for the asymmetric supercapacitor (Figure 6c) with only one set of fingers covered by 4-printing-layer graphene, its CV curves exhibit clear asymmetry. These prove the efficiency of our printed graphene for energy storage. Moreover, the reliable printing technology of graphene allows the direct integration with printed current collectors, which leads to low equivalent series resistance $(\sim 8.7 \Omega)$ and rapid frequency response (the resistance-capacitor time constant is $13 \mathrm{~ms}$, as compared with a typical value of $1 \mathrm{~s}$ for normal supercapacitors). ${ }^{[13]}$
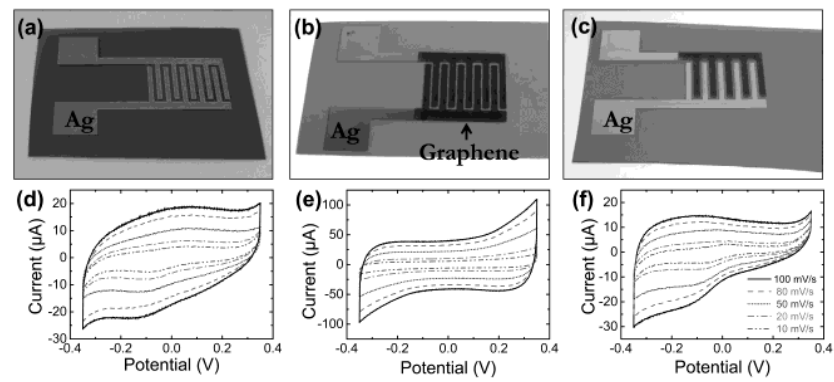

Figure 6. Inkjet printed interdigitated graphene supercapacitors on Kapton. (a) Printed silver current collectors. (b) Printed graphene electrodes (8 printing layers) on top of silver current collectors. (c) Asymmetric supercapacitors where only one set of silver figures is covered by printed graphene (4 printing layers). All the fingers are $1.0 \mathrm{~mm}$ wide, $9.4 \mathrm{~mm}$ long and interspaced by $0.6 \mathrm{~mm}$. (d) (e) and (f) are the CV curves measured in $1 \mathrm{M} \mathrm{Na}_{2} \mathrm{SO}_{4}$ aqueous solutions for (a), (b) and (c), respectively. Adapted with permission from Ref. [13].

$\mathrm{MoS}_{2}$-based photodetectors. Figure 7 shows printed $\mathrm{MoS}_{2}$ photodetectors on $\mathrm{SiO}_{2} / \mathrm{Si}$ wafers after annealing at $450{ }^{\circ} \mathrm{C}$ in $\mathrm{N}_{2}$ atmosphere. The electrodes (vertical strips in Figure 7a) are printed with commercial silver nanoparticle ink, and the channels (horizontal line in Figure 7a) are printed with our $\mathrm{MoS}_{2}$ ink. The SEM image (Figure 7b), Raman spectra (Figure 7c) as well as AFM analysis confirm the presence of $\mathrm{MoS}_{2}$ flakes with lateral dimension at the level of $100 \mathrm{~nm}$ and thickness of about 5-7 nm Even without specific engineering, these devices in such a simple structure have already been very responsive to the switching of any illumination ranging from ultraviolet light to infrared light. As shown in Figure $7 d$, the time-resolved current curves exhibit unique peaks (valleys) in response to illumination (dimming). The peaks and valleys likely result from the correlation between the intrinsic photoresponse of $\mathrm{MoS}_{2}$ and the trapping/detrapping process in the printed $\mathrm{MoS}_{2}$ films. ${ }^{[14]}$ In addition, the responsivity reaches the level of $40 \mu \mathrm{A} / \mathrm{W}$ and the response time is less than $0.6 \mathrm{~s}$. This performance is comparable to the $\mathrm{MoS}_{2}$ devices fabricated by the Langmuir-Blodggett method. ${ }^{[34]}$ It confirms again that our inkjet printing process retains the unique performance of the $2 \mathrm{D}$ materials.
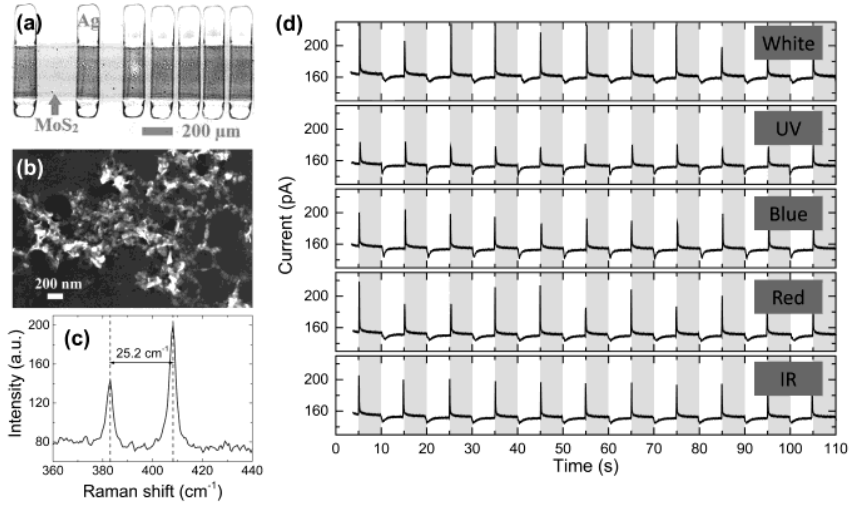

Figure 7. Inkjet printed $\mathrm{MoS}_{2}$ photodetectors on $\mathrm{Si} / \mathrm{SiO}_{2}$ wafers. (a) Optical micrograph of the printed devices. For passivation, the overall devices are covered by a 50 -nm-thick $\mathrm{Al}_{2} \mathrm{O}_{3}$ film fabricated by atomic layer deposition. (b) An SEM image of the printed film showing $\mathrm{MoS}_{2}$ flakes. (c) Raman spectra confirm the existence of $\mathrm{MoS}_{2}$. (d) Time-resolved current curves for the printed $\mathrm{MoS}_{2}$ devices under different illuminations: (from top to bottom) white, ultraviolet blue, red and infrared light. During measurement, the illumination is switched on (the shaded intervals) or off (the unshaded intervals) for every 5 seconds. Upon each switch on (off), there is a responsive peak (valley) in the current curves for all illuminations. Adapted with permission from Ref. [14].

Finally, it is worth mentioning that all the printed devices in this Concept rely on a high-temperature $\left(>400^{\circ} \mathrm{C}\right)$ annealing to remove the stabilizing polymers and recover the desired performance of 2D materials. The annealing, however, is not compatible with most plastic substrates used in flexible electronics. In order to extend the applications of our printing technology, low-temperature or selective sintering techniques well developed for printed metal nanoparticles might be incorporated, such as electrolyte sintering, ${ }^{[35]}$ "built-in" sintering, ${ }^{[36]}$ microwave sintering ${ }^{[37]}$ and laser sintering. ${ }^{[38]}$

\section{Conclusion}

The proposed technology concept is promising to overcome several great challenges in inkjet printing of the emerging $2 \mathrm{D}$ layered materials. Combining the distillation-assisted solvent exchange with polymer stabilization, we formulate stable, highconcentration, environmentally-friendly and printable inks for graphene and $\mathrm{MoS}_{2}$. The inks produce excellent jetting performance, and conformal and uniform printed patterns at high resolution of tens of micrometers. They may also offer a general strategy to suppress the well-known and often undesired coffeering effects in inkjet printing. More importantly, our printing 
technology allows fabricating electronic devices in a simple way (typically several printing passes followed by a simple annealing), but does not evidently diminish the unique properties of the 2D material nanosheets. We demonstrate that the inkjet printed transparent conductors, supercapacitors and photodetectors based on graphene or $\mathrm{MoS}_{2}$ have comparable or superior performance to those fabricated through other liquid-phase techniques. In conclusion, our inkjet printing technology for 2D layered materials has substantially improved the manufacturing efficiency and product quality. It is therefore very promising for emerging organic and printed electronics.

\section{Acknowledgements}

We gratefully acknowledge support and sponsorship by the European Research Council through the Advanced Investigator Grant OSIRIS (No. 228229), the Proof of Concept Grant iPUBLIC (No. 641416) and the Starting Grant InteGraDe (No. 307311), the Swedish Research Council through the grant iGRAPHENE, the Göran Gustafsson Foundation through the Young Researcher Prize (No. 1415B), as well as the German Research Foundation (LE 2440/1-1).

Keywords: inkjet printing - 2D layered materials - graphene $\mathrm{MoS}_{2} \cdot$ distillation assisted solvent exchange $\cdot$ printed electronics $\cdot$ coffee-ring effects

[1] B.-J. de Gans, P. C. Duineveld, U. S. Schubert, Adv. Mater. 2004, 16, 203-213.

[2] OE-A Roadmap for Organic and Printed Electronics, Organic Electronics Association, Frankfurt, 2011.

[3] D. Makarov, D. Karnaushenko, O. G. Schmidt, Chemphyschem 2013 14, 1771-1776.

[4] M. Singh, H. M. Haverinen, P. Dhagat, G. E. Jabbour, Adv. Mater 2010, 22, 673-685.

[5] J.-U. Park, M. Hardy, S. J. Kang, K. Barton, K. Adair, D. K Mukhopadhyay, C. Y. Lee, M. S. Strano, A. G. Alleyne, J. G. Georgiadis, P. M. Ferreira, J. A. Rogers, Nat. Mater. 2007, 6, 782789.

[6] T. Sekitani, Y. Noguchi, U. Zschieschang, H. Klauk, T. Someya, Proc Natl. Acad. Sci. U. S. A. 2008, 105, 4976-4980.

[7] T. H. J. van Osch, J. Perelaer, A. W. M. de Laat, U. S. Schubert, Adv Mater. 2008, 20, 343-345.

[8] J. Li, T. Unander, A. L. Cabezas, B. Shao, Z. Liu, Y. Feng, E. B Forsberg, Z.-B. Zhang, I. Jõgi, X. Gao, M. Boman, L.-R. Zheng, M. Östling, H.-E. Nilsson, S.-L. Zhang, J. Appl. Phys. 2011, 109, 084915.

[9] K. S. Novoselov, V. I. Fal'ko, L. Colombo, P. R. Gellert, M. G. Schwab, K. Kim, Nature 2012, 490, 192-200.

[10] Q. H. Wang, K. Kalantar-Zadeh, A. Kis, J. N. Coleman, M. S. Strano Nat. Nanotechnol. 2012, 7, 699-712.

[11] J. N. Coleman, M. Lotya, A. O'Neill, S. D. Bergin, P. J. King, U. Khan K. Young, A. Gaucher, S. De, R. J. Smith, I. V Shvets, S. K. Arora, G. Stanton, H.-Y. Kim, K. Lee, G. T. Kim, G. S. Duesberg, T. Hallam, J.
J. Boland, J. J. Wang, J. F. Donegan, J. C. Grunlan, G. Moriarty, A. Shmeliov, R. J. Nicholls, J. M. Perkins, E. M. Grieveson, K. Theuwissen, D. W. McComb, P. D. Nellist, V. Nicolosi, Science 2011, $331,568-571$.

S. Park, R. S. Ruoff, Nat. Nanotechnol. 2009, 4, 217-224

[13] J. Li, F. Ye, S. Vaziri, M. Muhammed, M. C. Lemme, M. Östling, Adv. Mater. 2013, 25, 3985-3992.

[14] J. Li, M. M. Naiini, S. Vaziri, M. C. Lemme, M. Östling, Adv. Funct. Mater. 2014, DOI: 10.1002/adfm.201400984. (Submitted)

[15] J. Li, F. Ye, S. Vaziri, M. Muhammed, M. C. Lemme, M. Östling Carbon 2012, 50, 3113-3116.

[16] Y. Hernandez, V. Nicolosi, M. Lotya, F. M. Blighe, Z. Sun, S. De, I. T. McGovern, B. Holland, M. Byrne, Y. K. Gun'Ko, J. J. Boland, P. Niraj, G. Duesberg, S. Krishnamurthy, R. Goodhue, J. Hutchison, V. Scardaci, A. C. Ferrari, J. N. Coleman, Nat. Nanotechnol. 2008, 3 563-568

[17] U. Khan, A. O'Neill, M. Lotya, S. De, J. N. Coleman, Small 2010, 6, 864-871

[18] U. Khan, A. O'Neill, H. Porwal, P. May, K. Nawaz, J. N. Coleman Carbon 2012, 50, 470-475

Y. T. Liang, M. C. Hersam, J. Am. Chem. Soc. 2010, 132, 17661 17663.

E. B. Secor, P. L. Prabhumirashi, K. Puntambekar, M. L. Geier, M. C. Hersam, J. Phys. Chem. Lett. 2013, 4, 1347-1351.

Z. Liu, D. D. L. Chung, J. Electron. Mater. 2004, 33, 1316-1325.

B. Kim, Y.-H. Lee, J.-H. Ryu, K.-D. Suh, Colloids Surf. APhysicochem. Eng. Asp. 2006, 273, 161-164.

E. B. Secor, S. Lim, H. Zhang, C. D. Frisbie, L. F. Francis, M. C. Hersam, Adv. Mater. 2014, DOI: 10.1002/adma.201401052.

X. Zhang, A. C. Coleman, N. Katsonis, W. R. Browne, B. J. van Wees, B. L. Feringa, Chem. Commun. 2010, 46, 7539-7541.

D. Soltman, V. Subramanian, Langmuir 2008, 24, 2224-2231.

R. D. Deegan, O. Bakajin, T. F. Dupont, G. Huber, S. R. Nagel, T. A Witten, Nature 1997, 389, 827-829.

M. Kuang, L. Wang, Y. Song, Adv. Mater. 2014, DOI: 10.1002/adma.201305416.

[29] M. Kuang, J. Wang, B. Bao, F. Li, L. Wang, L. Jiang, Y. Song, Adv Opt. Mater. 2014, 2, 34-38.

B.-J. de Gans, U. S. Schubert, Langmuir 2004, 20, 7789-7793.

P. J. Yunker, T. Still, M. A. Lohr, A. G. Yodh, Nature 2011, 476, 308311.

J. Park, J. Moon, Langmuir 2006, 22, 3506-3513.

[33] W. Gao, N. Singh, L. Song, Z. Liu, A. L. M. Reddy, L. Ci, R. Vajtai, Q. Zhang, B. Wei, P. M. Ajayan, Nat. Nanotechnol. 2011, 6, 496-500. 
[35] Y. Long, J. Wu, H. Wang, X. Zhang, N. Zhao, J. Xu, J. Mater. Chem. 2011, 21, 4875-4881.

[36] M. Grouchko, A. Kamyshny, C. F. Mihailescu, D. F. Anghel, S.

Received: ((will be filled in by the editorial staff))

Magdassi, ACS Nano 2011, 5, 3354-3359.

Published online: ((will be filled in by the editorial staff))

[37] J. Perelaer, B.-J. de Gans, U. S. Schubert, Adv. Mater. 2006, 18, 2101-2104.

[38] S. H. Ko, H. Pan, C. P. Grigoropoulos, C. K. Luscombe, J. M. J. Fréchet, D. Poulikakos, Nanotechnology 2007, 18, 345202. 
Entry for the Table of Contents (Please choose one layout)

Layout 1:

\section{CONCEPTS}

A simple and general solvent exchange technology is introduced for inkjet printing of 2D layered materials. The technology addresses the critical challenges and improves the manufacturing efficiency and product quality. We have demonstrated excellent jetting performance, ideal printing patterns and a variety of promising applications for inkjet printing of $2 \mathrm{D}$ layered materials.

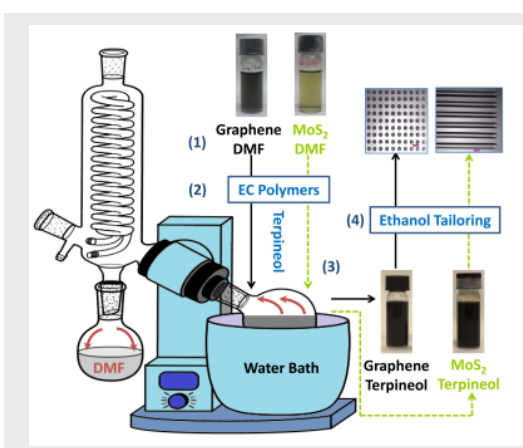

Jiantong Li, ${ }^{*}$ Max C. Lemme, and Mikael Östling

Page No. - Page No.

Inkjet Printing of 2D Layered

Materials

Layout 2:

\section{CONCEPTS}

$$
\begin{aligned}
& \text { ((Insert TOC graphic (.tiff, .eps, .pdf) here } \\
& >300 \text { dpi } \\
& 110 \mathrm{~mm} \times 25 \mathrm{~mm}
\end{aligned}
$$

((Author(s), Corresponding Author(s)*))

\section{Page No. - Page No.}

((Title))

((Text for Table of Contents

375-475 characters, including spaces

Start with catchy bold text)) 